\title{
AN ELEMENTARY PROOF OF SURJECTIVITY FOR A CLASS OF ACCRETIVE OPERATORS
}

\author{
WILLIAM O. RAY ${ }^{1}$
}

\begin{abstract}
An operator $\boldsymbol{A}$ defined on a real Banach space $X$ is said to be locally accretive if, for each $\lambda>0, x \in X$ and each $y$ near $x,\|x-y\|<\| x$ $-y+\lambda(A x-A y) \|$. It is shown that if $A: X \rightarrow X$ is locally accretive and locally Lipschitzian then $(I+A)(X)=X$.
\end{abstract}

Let $X$ be a real Banach space. A nonlinear operator $A: X \rightarrow X$ is said to be accretive if, for each $\lambda>0$ and for each $w, y \in X$,

$$
\|w-y\|<\|w-y+\lambda(A w-A y)\|,
$$

while $A$ is said to be locally accretive if (1) holds for all $\lambda>0$ when $y$ is sufficiently near $w$.

The nonlinear accretive operators were introduced independently in 1967 by F. E. Browder [3] and T. Kato [9], and they observed that $A$ is accretive if and only if $j(A w-A y)>0$ for some $j \in X^{*}$ satisfying $\|j\|=\|w-y\|$ and $j(w-y)=\|w-y\|^{2}$. An early fundamental result in the theory of accretive operators, due to Browder [4], states that the initial value problem

$$
\frac{d u}{d t}+A u=0, \quad u(0)=w
$$

is solvable when $A$ is locally Lipschitzian and accretive on $X$, a result which was subsequently generalized by $R$. H. Martin [12] to the continuous accretive operators. The existence theory for equation (2), which is quite technical and complex, has since been generalized to cases in which $A$ is neither continuous nor single valued (see, for example, [11], [13] or [14]).

Utilizing the existence results for (2), Browder [4] and later Martin [12] showed that if $\boldsymbol{A}$ is locally Lipschitzian (in Martin's theorem only continuity is required) and accretive, then $A$ is m-accretive, i.e., $(I+A)(X)=X$. Recently M. Crandall and A. Pazy [7] have given a proof of Martin's result which does not rely on solvability of (2), but which remains rather complex, being based on an intricate iteration scheme. It is our purpose in this note to present an elementary and straightforward proof of Browder's theorem for locally accretive operators.

Received by the editors November 10, 1977.

AMS (MOS) subject classifications (1970). Primary 47H15, 47H10.

Key words and phrases. Locally accretive, $m$-accretive.

${ }^{1}$ Research carried out in part while the author was a student at the University of Iowa. 
Theorem 1. Let $X$ be a real Banach space and let $A: X \rightarrow X$ be a locally accretive operator. Suppose $A$ satisfies the following local Lipschitz condition:

For each $w \in X$ there is an $\epsilon>0$ and an $M>0$ such that $\|A x-A y\|<M\|x-y\|$ whenever $\|x-w\|<\epsilon$ and $\|w-y\|<\epsilon$.

Then $A$ is $m$-accretive.

We will use the following fixed point theorem, due to J. Caristi.

TheOReM C [5]. Let $(M, d)$ be a complete metric space, g: $M \rightarrow M$ an arbitrary mapping and $\varphi: M \rightarrow[0, \infty)$ a lower semicontinuous mapping. Suppose for all $x \in M$ that $g$ satisfies

$$
d(g(x), x)<\varphi(x)-\varphi(g(x)) .
$$

Then $g$ has a fixed point in $M$.

The above theorem is essentially equivalent to a theorem of I. Ekeland [8], which is in turn an abstraction of a lemma due to Bishop and Phelps [1]. Since its appearance in 1976, this theorem has found numerous applications in varied areas (see [2] or [10] for example). In this note we continue this trend by using the theorem to substantially simplify known proofs of results similar to our Theorem 1 . It is our hope that, by pointing out a connection between Theorem $\mathbf{C}$ and the accretive operators, other of the results alluded to above may also ultimately find similar simplification.

Proof of Theorem 1. Note that the operator $A_{y}: X \rightarrow X$ given by $A_{y}(x)=$ $A x-y$ satisfies the hypotheses of the theorem and that $0 \in\left(I+A_{y}\right)(X)$ if and only if $y \in(I+A)(X)$; thus it suffices to show that if $A: X \rightarrow X$ is locally accretive and satisfies (3) then $0 \in(I+A)(X)$.

We begin by showing that for each $w \in X$ there is an $x \in X$ arbitrarily near to $w$ and a $\lambda>1$ such that

$$
\lambda x+(\lambda-1) A x=w .
$$

For $w \in X$ and $\epsilon>0$ choose $M>0$ and $\hat{\epsilon} \in(0, \epsilon)$ in accordance with (3). Set $D=\{x \in X:\|w-x\|<\hat{\epsilon}\}$ and choose $r \in(0,1)$ so small that $r M<1$ and $r\|w+A x\|<\hat{\epsilon}$ for all $x \in D$. Define $f(x)=(1-r) w-r A x$. Since, for $x, y \in D$,

$$
\|f(x)-f(y)\|=r\|A y-A x\|<r M\|x-y\|
$$

and

$$
\|f(x)-w\|=r\|w+A x\|<\hat{\epsilon}
$$

we conclude that $f$ is a contraction mapping of $D$ into itself. Thus there is an $x \in D$ such that $f(x)=x$, and hence

$$
w=(1-r)^{-1} x+r(1-r)^{-1} A x
$$

which establishes (4).

Now for $w \in X$, choose $\epsilon$ so small that (1) holds whenever $\|w-y\|<\epsilon$. 
Then there exists $x \in X$ and $\lambda>1$ so that $\|x-w\|<\epsilon$ and $\lambda x+(\lambda-1) A x$ $=w$. Since $w-x=(\lambda-1)(x+A x)$, we have (using (1)):

$$
\begin{aligned}
\|w+A w\| & =\|\lambda x+(\lambda-1) A x+A w\| \\
& =\|\lambda(x+A x)+A w-A x\| \\
& =\left\|\lambda(\lambda-1)^{-1}(w-x)+A w-A x\right\| \\
& \geqslant\left\|\lambda(\lambda-1)^{-1}(w-x)\right\| \\
& =\lambda\|x+A x\|,
\end{aligned}
$$

i.e., $\lambda\|x+A x\| \leqslant\|w+A w\|$. Now define $g: X \rightarrow X$ by $g(w)=x$. Then

$$
\begin{aligned}
\|g(w)-w\| & =\|x-(\lambda x+(\lambda-1) A x)\| \\
& =\lambda\|x+A x\|-\|x+A x\| \\
& <\|w+A w\|-\|g(w)+A g(w)\|,
\end{aligned}
$$

and an application of Theorem $\mathrm{C}$ (with $\varphi(w)=\|w+A w\|)$ implies that $g$ has a fixed point $w \in X$, i.e., $w=\lambda w+(\lambda-1) A w$. Since $\lambda>1$, this implies $0 \in(I+A) X$ and the theorem is proved.

Remarks. (1) The local Lipschitz condition (3) is used only to obtain solvability of the equation (4); we do not know if there is a direct proof that equation (4) is solvable for accretive (or locally accretive) operators which are assumed only to be continuous. We note that, in case $A$ is not continuous, conditions similar to (4) have been used to obtain solutions to the problem (1) (see, for example, [6]).

(2) The referee to this paper has pointed out that it is an easy exercise to show that "locally accretive" operators are actually "globally accretive" and thus that our theorem is equivalent to Browder's result [4]. As we have already noted however, our application of Theorem $\mathbf{C}$ permits a significant simplification in the proof.

\section{REFERENCES}

1. E. Bishop and R. R. Phelps, The support functionals of a convex set, Proc. Sympos. Pure Math., vol. 7, Amer. Math. Soc., Providence, R. I., 1963, pp. 27-36.

2. H. Brezis and F. E. Browder, A general principle on ordered set in nonlinear functional analysis, Advances in Math. 21 (1976), 777-787.

3. F. E. Browder, Nonlinear mappings of nonexpansive and accretive type in Banach spaces, Bull. Amer. Math. Soc. 73 (1967), 875-882.

4. Nonlinear monotone and accretive operators in Banach spaces, Proc. Nat. Acad. Sci. U.S.A. 61 (1968), 388-393.

5. J. Caristi, Fixed point theorems for mappings satisfying inwardness conditions, Trans. Amer. Math. Soc. 215 (1976), 241-251.

6. M. Crandall and T. Liggett, Generation of semigroups of nonlinear transformations on general Banach spaces, Amer. J. Math. 93 (1971), 265-298.

7. M. Crandall and A. Pazy, On the range of accretive operators, Israel J. Math. 27 (1977), 235-246.

8. I. Ekeland, On the variational principle, J. Math. Anal. Appl. 47 (1974), 17-23.

9. T. Kato, Nonlinear semigroups and evolution equations, J. Math. Soc. Japan 19 (1967), 508-520. 
10. W. A. Kirk, Caristi's fixed point theorem and metric convexity, Colloq. Math. J. 35 (1976), 81-86.

11. Y. Kobayashi, Difference approximation of Cauchy problems for quasidissipitive operators and generation of nonlinear semigroups, J. Math. Soc. Japan 27 (1975), 640-665.

12. R. H. Martin, Jr., A global existence theorem for autonomous differential equations in Banach space, Proc. Amer. Math. Soc. 26 (1970), 307-314.

13. M. Pierre, Un théorème général de génération de semi-groupes non-linéaires, Israel J. Math. 23 (1976), 189-199.

14. Génération et perturbation de semi-groupes de contractions non-linéaires, Thèse de Docteur de 3è cycle, Univ. de Paris VI, 1976.

Departmient of Mathematics, Iowa State University, Ames, Iowa 50011 